\title{
Investigation on Reaction Diffusion Process Inside a Porous Bio-Catalyst Using DTM
}

\section{Azimi $\mathbf{M}^{1}$ and Azimi $\mathbf{A}^{2 *}$}

${ }^{1}$ Faculty of New Sciences and Technologies, University of Tehran, Tehran, Iran

${ }^{2}$ Department of Chemical Engineering, College of Chemical Engineering, Mahshahr Branch, Islamic Azad University, Mahshahr, Iran

\begin{abstract}
In this paper, the reaction-diffusion problem in a biocatalyst has been investigated analytically. The efficiency of Differential Transformation Method (DTM) has been demonstrated by solving a problem of chemical/ bio chemical engineering namely, reaction-diffusion process inside a porous biocatalyst in which occurring biochemical reaction follows Michaelis-Menten kinetics. Also the numerical method is used for the validity of this analytical method and excellent agreement is observed between the solutions obtained from DTM and numerical results. Trusting this validity, effects of some other parameters are discussed.
\end{abstract}

Keywords: Biocatalyst, Reaction-diffusion, Analytical solution, DTM

\section{Nomenclature}

Concentration of inside the

$C_{A}$ catalyst pores

Greek Letters

Concentration at the catalyst

$C_{\$}$ surface

Effective diffusivity of species

$D_{e} \quad A$

Parameter in Michaelis-Menten

$K_{m}$ kinetics

Coordinate in the radial

$r$ direction

Parameter in Michaelis-Menten

$r_{m} \quad$ kinetics

$R \quad$ Particle radius

$u$ Dimensionless Concentration

\section{Introduction}

The prediction of diffusion and reaction rates in porous catalyst is an important problem in chemical engineering, indeed when the reaction rate depends on concentration in a nonlinear case. The model for diffusion and reaction inside a porous biocatalyst has been discussed extensively by pervious researchers $[1,2]$.

The concept of effectiveness factor is an important one in heterogeneous catalysis and in solid fuel. The effectiveness factor is widely used to account for the interaction between pore diffusion and reactions on pore walls in porous catalytic pellets and solid fuel particles. The effectiveness factor is defined as the ratio of the reaction rate actually observed to the reaction rate calculated if the surface reactant concentration persisted throughout the interior of the particle, that is, no reactant concentration gradient within the particle. The reaction rate in a particle can therefore be conveniently expressed by its rate under surface conditions multiplied by the effectiveness factor. This concept was first developed mathematically by Thiele [3], and has since been extended by many other workers. Extensive investigation of analytical solutions and methods for the approximation of the effectiveness factor can be found in Aris [4,5]. The state of development of the theory up till the last decade has been summarized by Wijngaarden et al. [6].
In the heart of all the different engineering sciences, everything shows itself in the mathematical relation that most of these problems and phenomena are modeled by ordinary or partial differential equations. In most cases, scientific problems are inherently of nonlinearity that does not admit analytical solution, so these equations should be solved using special techniques [7-9].

\section{Problem Description}

Consider an inert permeable spherical solid particle. Bacterial immobilization has been done inside the porous particle by usual methods, and these porous particles with bacteria immobilized inside it which is termed as biocatalyst. This biocatalyst is immersed in a pool of liquid containing the substrate. It is assumed that the resistance due to film surrounding the biocatalyst is negligibly small under the operating conditions in the bioreactor. Thus, the substrate $A$ diffuses inside biocatalyst and the biochemical reaction occurs therein simultaneously. The component mass balance for the substrate over a thin spherical shell inside the biocatalyst yields the following model equation with the associated Boundary Conditions [10].

$$
\begin{aligned}
& D_{e}\left(\frac{d^{2} C_{A}}{d r^{2}}+\frac{2}{r} \frac{d C_{A}}{d r}\right)=\frac{r_{m} C_{A}}{K_{m}+C_{A}} \\
& r=R \rightarrow C_{A}=C_{A S}, \quad r=0 \rightarrow \frac{d C_{A}}{d r}=0
\end{aligned}
$$

Where $C_{A}$ is concentration of species $A$ inside the catalyst pores, $C_{A S}$ concentration of species $A$ at the catalyst surface, $D_{e}$ is diffusion coefficient of species $K_{m}$ inside the catalyst pores, $K_{m}$ is parameter in Michaelis-Mentn kinetics, $r$ is coordinate in the radial direction and $r_{m}$ is parameter in Michaelis-Menten kinetics.

*Corresponding author: Azimi A, Department of Chemical Engineering, College of Chemical Engineering, Mahshahr Branch, Islamic Azad University, Mahshahr, Iran, Tel: +91240-240311; E-mail: meysam.azimi@gmail.com

Received February 13, 2015; Accepted March 24, 2015; Published April 04, 2015

Citation: Azimi M, Azimi A (2015) Investigation on Reaction Diffusion Process Inside a Porous Bio-Catalyst Using DTM. J Bioequiv Availab 7: 123-126. doi:10.4172/jbb.1000225

Copyright: ( 2015 Azimi M, et al. This is an open-access article distributed under the terms of the Creative Commons Attribution License, which permits unrestricted use, distribution, and reproduction in any medium, provided the original author and source are credited. 
in above model equation, it s assumed that the biochemical reaction follows Michaelis-Menten kinetics. The derivation of Equation may be found in an excellent text book by Fogler [11]. Introducing the following dimensionless variables [12]:

$$
u=\frac{C_{A}}{C_{A S}}, \eta=\frac{r}{R}, \phi=\sqrt{\frac{\left(-r_{A S}\right) R^{2}}{D_{e} C_{A S}}}=\sqrt{\frac{R^{2} r_{m}}{D_{e} K_{m}(1+\beta)}}
$$

Here, $\varphi$ is the Thiele modulus and it signifies the ration of the intrinsic chemical reaction rate in the absence of mass transfer limitation to the rate of diffusion through the catalyst, i.e.

$$
\phi^{2}=\frac{(\text { reaction rate })_{\text {catalyst surface }}}{(\text { diffusion rate })_{\text {catalyst sufface }}}
$$

Rewriting equation using equation yields:

$$
\begin{aligned}
& \frac{d^{2} u}{d \eta^{2}}+\frac{2}{\eta}-\phi^{2} \frac{(1+\beta) u}{(1+\beta u)}=0, \\
& \eta=1 \rightarrow u=1, \quad \eta=0 \rightarrow \frac{d u}{d \eta}=0
\end{aligned}
$$

As it has been mentioned in boundary condition, the concentration of species $A$ is maximum at the catalyst surface.

\section{Basic Idea of Differential Transformation Method}

Let $x(t)$ be analytic in a domain $D$ and let $t=t_{i}$ represents any point in $D$. The function $x(t)$ is then represented by one power series whose center is located at $t_{i}$. The Taylor series expansion function of $x(t)$ is in the form of:

$$
x(t)=\sum_{k=0}^{\infty} \frac{\left(t-t_{i}\right)}{k !}\left[\frac{d^{k} x(t)}{d t^{k}}\right]_{t=t_{i}} \quad \forall t \in D
$$

As explained in [4] the differential transformation of the function $x(t)$ is defined as follows:

$$
X(k)=\sum_{k=0}^{\infty} \frac{H^{k}}{k !}\left[\frac{d^{k} x(t)}{d t^{k}}\right]_{t=0}
$$

Where $x(t)$ the original is function and $X(k)$ is the transformed function. The differential inverse transform of $X(k)$ is defined as follows:

$$
x(t)=\sum_{k=0}^{\infty}\left(\frac{t}{H !}\right)^{k} X(k)
$$

Mathematical operations performed by DTM are listed in Table 1.

\section{Application of Differential Transformation Method}

In order to solve equation.4, we consider the following boundary conditions:

$$
u(0)=a, u^{\prime}(0)=0
$$

Where, $a$ should be determined.

Taking differential transform of equation.7 by using the related definitions in Table 1, we obtain:

$$
\begin{aligned}
& (k+1)(k) U(k+1)+\beta \sum_{s=0}^{k} \sum_{m=0}^{k-s} U(k-m+1)(k-s+1)(k-s)(k-s+1)+2(k+1) U(k+1)+ \\
& 2 \beta \sum_{l=0}^{k} U(l)(k-l+1) U(k-l+1)-\alpha U(k-1)
\end{aligned}
$$

As mentioned before in equation. 8, we can write:

$$
\begin{aligned}
& U(0)=a \\
& U(1)=0
\end{aligned}
$$

We can easily yield:

$$
\begin{aligned}
& U(2)=-\frac{\alpha a}{6-4 \beta-4 \beta a} \\
& U(3)=-\frac{22 \beta \alpha a}{(6-4 \beta-4 \beta a)(12-22 \beta-6 \beta \alpha)} \\
& \begin{aligned}
& U(4) \\
&\left(\begin{array}{l}
660 \beta^{2} a \alpha+1386 \beta^{2} \alpha a^{2}+88 \beta^{3} \alpha a-308 \alpha a^{2}-396 \alpha a^{3} \beta^{3}-45 \alpha^{2} a \beta-9 \alpha^{2} a^{2} \beta \\
+6 \beta^{2} \alpha^{2} a^{2}+22 \beta^{2} \alpha^{2} a+18 \alpha^{2} a-1188 \beta \alpha a
\end{array}\right) \\
&\left(\begin{array}{l}
-36736 a \beta^{3}-14576 a^{2} \beta^{3}+14704 a \beta^{4}+11120 a^{2} \beta^{4}+2768 a^{3} \beta^{4}+192 a^{4} \beta^{4}-14400 \beta \\
+30180 \beta^{2}-23600 \beta^{3}+6160 \beta^{4}+2160-4824 a \beta+25236 a \beta^{2}+3984 a^{2} \beta^{2}
\end{array}\right)
\end{aligned}
\end{aligned}
$$

We avoid listing the other components. However it can be yield that the closed form of the solutions is:

$$
F(t)=F(0) \times t^{0}+F(1) \times t^{1}+F(2) \times t^{2}+\cdots
$$

Consider boundary condition $u(1)=1$, the coefficient of the highest power of $t$ requires being zero. Therefore $a$ can be easily yield.

\section{Results and Discussions}

Thiele modulus gives Relation between catalytic activity and size of particle. The Thiele Modulus was developed to describe the relationship between diffusion and reaction rate in porous catalyst pellets with no mass transfer limitations. This value is generally used in determining the effectiveness factor for catalyst pellets. For 9th-order irreversible reactions.

Validity of the differential transformation method is shown in Figure 1. Excellent agreement between the numerical and analytical solutions is obvious in Figure 1.

This accuracy gives us high confidence in validity of this problem and reveals an excellent agreement of engineering accuracy. This Investigation is completed by depicting the effects of some important parameters to evaluate how these parameters influence this fluid.

Figures $2 \mathrm{a}-2 \mathrm{c}$ displays the effects of Thiele modulus on dimensionless concentration for three numerical cases of $\beta=1, \beta=3$ and $\beta=5$ As the Thiele modulus increases the concentration decreases.

The effectiveness factor $\chi$ which is relevant in the design of biochemical reactors is defined as follows:

$$
\chi=\frac{\text { actual reaction rate within catalyst pores }}{\text { reaction rate at the surface conditions }}
$$

\begin{tabular}{|l|l|}
\hline Original function & transformed function \\
\hline$x(t)=\alpha f(t) \pm \beta g(t)$ & $X(k)=\alpha F(k) \pm \beta G(k)$ \\
\hline$x(t)=f(t) g(t)$ & $X(k)=\sum_{l=0}^{k} F(l) G(k-l)$ \\
$\mathrm{x}(\mathrm{t})=\frac{\mathrm{df}(\mathrm{t})}{\mathrm{dt}}$ & $X(k)=(k+1) F(k+1)$ \\
$x(t)=\frac{d^{2} f(t)}{d t^{2}}$ & $X(k)=(k+1)(k+2) F(k+2)$ \\
\hline
\end{tabular}

Table 1: Some of the basic operations of DTM. 


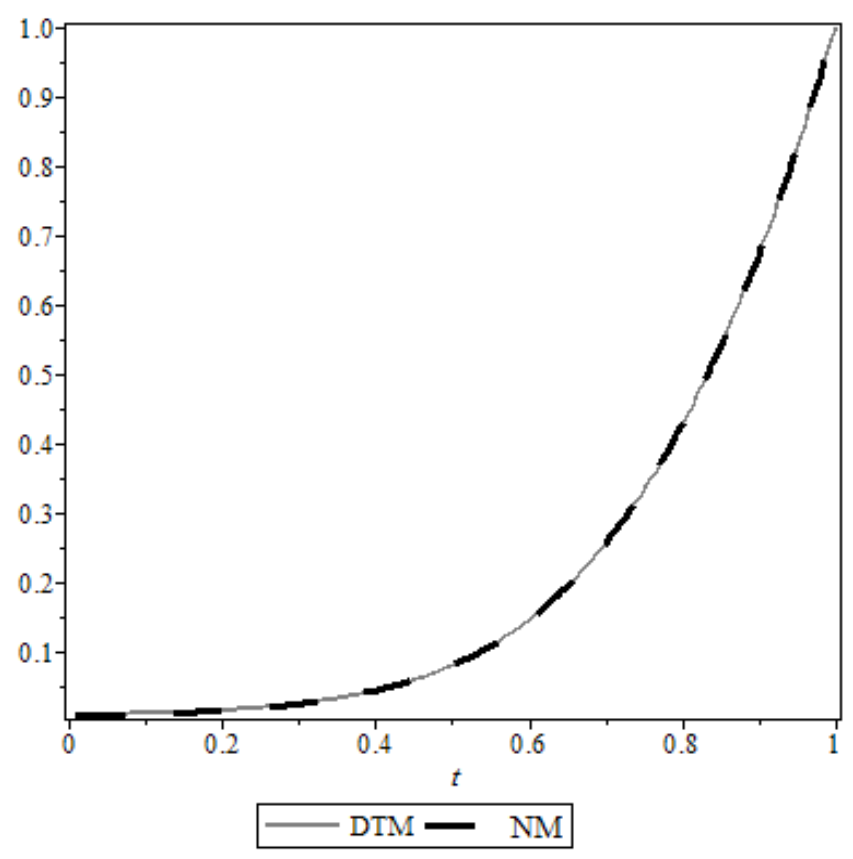

Figure 1: Comparison between DTM and Runge Kutta in case $\varphi=4, \beta=4$.

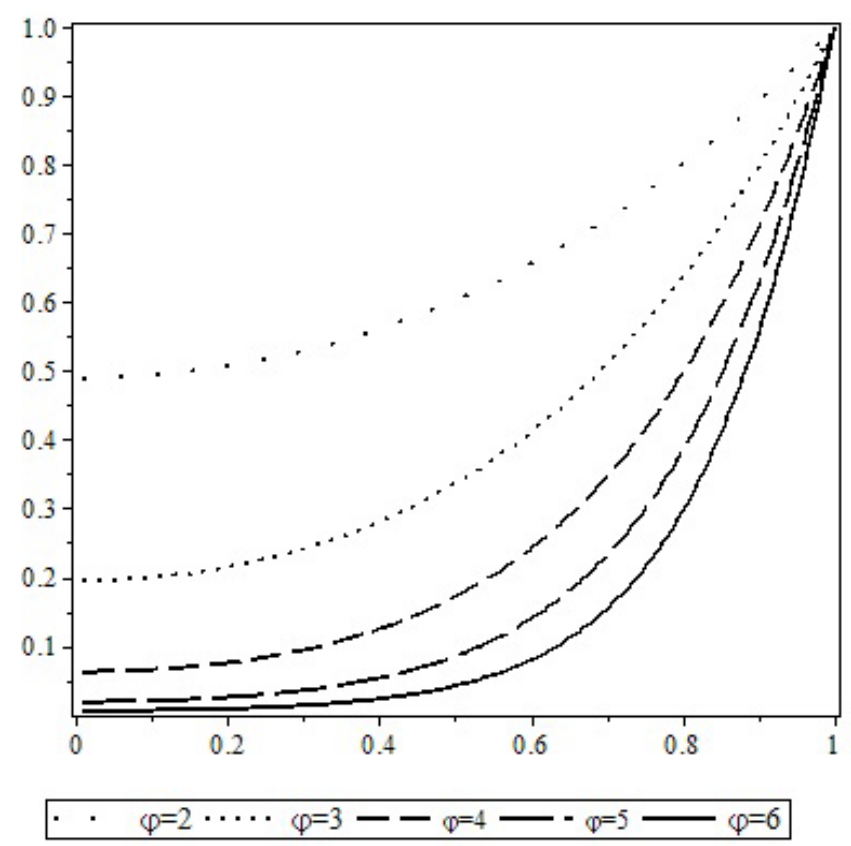

Figure 2a: Dimensionless concentration for various value of Thiele Modulus $\varphi$ in case $\beta=1$

According to [2], the above definition can mathematically be expressed in dimensionless form as follows:

$$
\chi=\left.\frac{3}{\phi^{2}} \frac{d u}{d \eta}\right|_{\eta=1}
$$

The effectiveness factor $\chi$ for various values of $\phi$ and $\beta$ is tabulated in Table 2.

\section{Conclusions}

In this paper, approximate analytical solution of radiation diffusion problem in a porous biocatalyst and indicated what these solutions mean in terms of the relative importance of the various Thiele modulus on this kind of problem, have been presented. Complementary numerical solutions were on obtained via forth order Runge Kutta

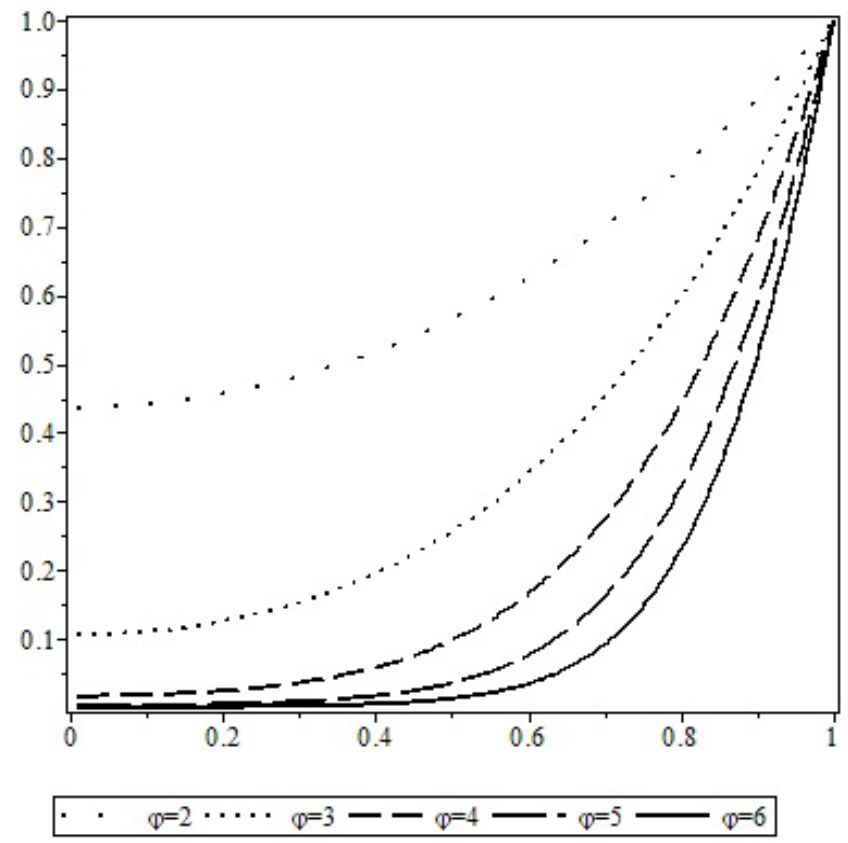

Figure 2b: Dimensionless concentration for various value of Thiele Modulus $\varphi$ in case $\beta=3$.

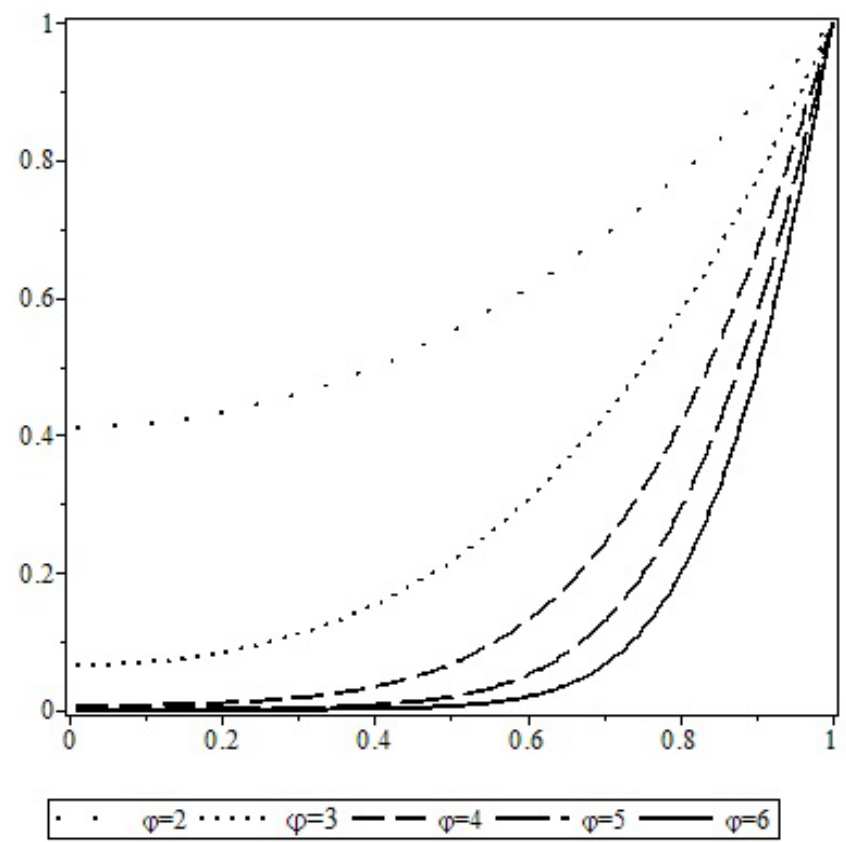

Figure 2c: Dimensionless concentration for various values of Thiele Modulus $(\varphi)$ in case $\beta=5$. 
Citation: Azimi M, Azimi A (2015) Investigation on Reaction Diffusion Process Inside a Porous Bio-Catalyst Using DTM. J Bioequiv Availab 7: 123126. doi: $10.4172 / \mathrm{jbb} .1000225$

\begin{tabular}{|c|c|c|}
\hline \multirow{3}{*}{$\beta$} & $\varphi$ & $\chi$ \\
\hline \multirow{3}{*}{1} & 2 & 0.871564 \\
\cline { 2 - 3 } & 4 & 0.626706 \\
\hline \multirow{3}{*}{2} & 6 & 0.463680 \\
\cline { 2 - 3 } & 2 & 0.902231 \\
\hline \multirow{3}{*}{3} & 4 & 0.659046 \\
\hline & 6 & 0.487476 \\
\hline & 2 & 0.925301 \\
\hline
\end{tabular}

Table 2: Value of effectiveness factor for various values of $\varphi$ and $\beta$.

and a very excellent agreement between the solutions obtained from DTM and the computations was observed. The effectiveness factor has been also achieved for various parameters. The results are in good agreement with the existing numerical results and therefore elucidate the reliability and efficiency of DTM. The comparisons made suggest that the DTM could be a useful and effective tool in solving systems of nonlinear differential equations of reaction diffusion process in a porous biocatalyst.

In brief,

The effectiveness factor $\chi$ values increases monotonically as the Thiele modulus $(\phi)$ increases.

The concentration inside the pores significantly rise and profiles move closer to surface as $\phi$ decrease.

$\beta$ has moderate influence on the effectiveness factor.

\section{References}

1. Li X, Chen XD, Chen N (2004) A third-order approximate solution of the reaction-diffusion process in an immobilized biocatalyst particle. Biochemical Engineering Journal 17: 65-69.

2. Lee J, Kim DH (2013) Global approximations of unsteady-state adsorption, diffusion, and reaction in a porous catalyst. AIChE Journal 59: 2540-2548.

3. Thiele EW (1939) Relation between catalytic activity and size of particle. Ind Eng Chem 31: 916-920

4. Aris $R$ (1975): The mathematical theory of diffusion and reaction in permeable catalyst I. Oxford: Clarendon Press, New York, USA

5. Aris $R$ (1975): The mathematical theory of diffusion and reaction in permeable catalyst II. Oxford: Clarendon Press,New York, USA

6. Wijngaarden RJ, Kronberg AE, Westerterp KR (1998) Industrial catalysis: Optimizing catalyst and processes. Weinheim: Wiley- $\mathrm{VCH}$.

7. Azimi M, Azimi A,Mirzaei M (2014) MHD Jeffery Hamel Problem with Graphene Oxide Nanoparticles: Analytical Investigation, Graphene2: 57-61.

8. Azimi M, Azimi A, Mirzaei M (2014) Investigation of the unsteady graphene oxide nanofluid flow between two moving plates. Journal of Computational and Theoretical Nanoscience 11: 2104-2108.

9. Azimi M, Azimi A, Mirzaei M (2015) Analytical Investigation of MHD Jeffery Hamel Problem with Graphene Oxide Nanoparticles Using GOHAM. Journal of Computational and Theoretical Nanoscience 12: 991-995.

10. Danish M, KumarSh, Kumar S (2010) Analytical solution of the reaction diffusion process in a permeable spherical catalyst.Chemical Engineering Technology 33: 664-675.

11. Shi-Bin L, Yan-Ping S, Scott K (2003) Analytic solution of diffusion reaction in spherical porous catalyst.Chemical Engineering Technology 26: 87-95.

12. Gottifredi JC, Gonzo EE (2005) On the effectiveness factor calculation for a reaction-diffusion process in an immobilized biocatalyst pellet. Biochemical Engineering Journal 24: 235-242. 